\title{
Color Image Transmission in Single-User Digitally Precoded Mmwave Non-Contiguous Orthogonal Frequency Division Multiplexing Wireless Communication System
}

\author{
Shammi Farhana Islam, Joarder Jafor Sadique², Jinia Rahman ${ }^{3}$, Shaikh Enayet Ullah ${ }^{3}$ \\ ${ }^{1}$ Department of Material Science and Engineering, University of Rajshahi, Rajshahi, Bangladesh \\ ${ }^{2}$ Department of Electronics and Telecommunication Engineering, Begum Rokeya University, Rangpur, Bangladesh \\ ${ }^{3}$ Department of Applied Physics and Electronic Engineering, University of Rajshahi, Rajshahi, Bangladesh
}

Email address:

sfi_mse@ru.ac.bd (S. F. Islam), joarder@brur.ac.bd (J. J. Sadique), jinia4944@gmail.com (J. Rahman), enayet_apee@ru.ac.bd (S. E. Ullah)

\section{To cite this article:}

Shammi Farhana Islam, Joarder Jafor Sadique, Jinia Rahman, Shaikh Enayet Ullah. Color Image Transmission in Single-User Digitally Precoded Mmwave Non-Contiguous Orthogonal Frequency Division Multiplexing Wireless Communication System. Advances in Wireless Communications and Networks. Vol. 3, No. 6, 2017, pp. 90-98. doi: 10.11648/j.awcn.20170306.13

Received: November 19, 2017; Accepted: December 15, 2017; Published: January 8, 2018

\begin{abstract}
Non-contiguous orthogonal frequency division multiplexing (NC-OFDM) has been considered as an outstanding technique for high data rate $5 \mathrm{G}$ and beyond $5 \mathrm{G}$ wireless communications. In this paper, we have made comprehensive performance evaluative study for a single user NC-OFDM scheme implemented wireless communication system under consideration of millimeter-wave (mmWave) large MIMO antenna configuration. In our $32 \times 256$ uniform linear antenna (ULA) configured simulated system under investigation, three selective modern channel coding (LDPC, Repeat and Accumulate and (3, 2) SPC) with a single higher order digital modulation (256-QAM)) and three signal detection (ZF, MMSE and LR-based linear detection) techniques have been utilized. On consideration of color image transmission in AWGN and Rayleigh fading channel, it is observable from MATLAB based simulation study that the Repeat and Accumulate channel encoded simulated system is very much robust and effective in retrieving color image under utilization of MMSE signal detection technique.
\end{abstract}

Keywords: NC-OFDM, Digital Precoding, Mmwave Geometrical Channel, SNR

\section{Introduction}

In perspective of meeting up challenges for developing high spectral and energy efficient $5 \mathrm{G}$ radio system, it is noticeable that the present research trend is focusing on new and novel multicarrier transmission techniques using noncontiguous subcarriers such as NC-OFDM, its enhanced version, Generalized Multicarrier (GMC) multiplexing, or its special case, namely the Non-contiguous Filter-Bank Multi-Carrier (NC-FBMC) technique. The NC-OFDM has been recognized as one of the suitable candidates for future Fifth Generation (5G) communication with cognitive capabilities. In cognitive radio literature, the NC-OFDM technique has been proposed in which the Fourier transform is used to provide orthogonally between subcarriers and a cyclic prefix (CP) with temporal length greater than channel delay spread is added to each NC-OFDM symbol to mitigate inter symbol interference. With possessing of sufficient spectral agility, the novel NC-OFDM technique is capable of facilitating simultaneous data transmission from unlicensed-system users (Secondary Users (SUs)) across several fragmented unoccupied frequency bands even in the presence of licensed-system users (Primary Users (PUs)) signals with increased spectrum utilization $[1,2]$. The NC-OFDM based cognitive radio (CR) system has the drawbacks of high side lobe power and peak-to-average power ratio (PAPR) values. A significant amount of work has already been done for minimization of such system drawbacks. However, In 1915, Hao and Lai proposed a precoding scheme to jointly reduce PAPR values in the secondary users (SUs) spectrum band and suppressed side lobes of signals in the primary user (PU) band [3]. In 2017, Elahi and et.al., considered NC-OFDM as an appropriate candidate for Cognitive radio (CR) physical layer and focused on its high out of band (OOB) radiation due to high side lobes. They proposed two algorithms for enhancement of spectrum 
sharing capability such as Genetic Algorithm (GA) and Firefly algorithm (FFA) for cancelling OOB radiation with inserting Cancellation subcarriers (CCs) on either side of the used NC-OFDM signal [4]. In 2017, Fathima and et.al., mentioned that the side-lobe suppression methods implemented in NC-OFDM based cognitive radio (CR) system with utilization of the fixed length rectangular windowing functions for canceling carriers (CC's) like Extended Active interference cancellation (EAIC) and active interference cancellation (AIC) methods, the CCs in different frequency had non-uniform assignment for side lobe suppression. To overcome such problem, they proposed a novel variable basis function in which the $\mathrm{CCs}$ are grouped by frequency positions and modeled with different waveforms of different length to suppress NC-OFDM side lobes effectively while reducing inter carrier interference (ICI) at the same time [5]. In addition to reviewing of non cognitive radio literature, it has been known that the $5 \mathrm{G}$ network has not yet been deployed commercially and also its physical layer technology and radio spectrum have not been standardized. There are predominantly three key approaches to address the $5 \mathrm{G}$ Network architectural designing such as (i) ultra-dense networks (UDNs): the network densification already has been adopted in existing $4 \mathrm{G}$ wireless cellular networks, which is essentially known as small cell technology, and a denser network can further boost the network capacity; (ii) large quantities of new bandwidth: migrating toward higher frequencies will release a large amount of bandwidth available to achieve higher capacity. In particular, the millimeter-wave ("mm Wave," for carrier frequencies of $30-300 \mathrm{GHz}$ ) communications can be the promising candidate and (iii) high spectrum efficiency: by using a large number of antennas (100 or more), massive multiple-input multiple-output (MIMO) can significantly improve the spectrum efficiency by extensively harnessing the available space resources [6]. In this present study, we have presented simulation results for Non-Contiguous Orthogonal Frequency Division Multiplexing Wireless Communication System under consideration of large MIMO antenna configuration and signal transmission with mmWave carrier frequency.

\section{Signal Processing Techniques}

In our present study various signal processing schemes have been used. A brief overview of these schemes is given below-

\subsection{Non-Contiguous Orthogonal Frequency-Division Multiplexing}

In NC-OFDM scheme, 256-sized IFFT block is considered where; the total number of subcarriers is 256 . Out of 256 subcarriers, merely 142 subcarriers are treated as active subcarriers for utilizing 142 complex digitally modulated data symbols. The remaining subcarriers are null subcarriers viz. they do not carry any data symbols. All the subcarrier indices are in the range: $\{-128,-127,-126 \ldots \ldots . .0,1,2,3, \ldots \ldots 127\}$ and the active subcarriers indices, IDC $=\{-100, \ldots,-62\} \cup$ $\{-41, \ldots,-11\} \cup\{10, \ldots, 40\} \cup\{61, \ldots, 101\}$. In this
NC-OFDM scheme, no cancellation carriers have been inserted. The 142 data symbols and the remaining 114 zeros are fed into IFFT block for multicarrier modulation [1]

\subsection{MIMO Fading Channel Estimation}

In estimation of ray path geometry based $8 \times 64$ sized mmWave MIMO fading channel $\mathbf{H}$, it is assumed that the $\mathrm{N}_{\mathrm{t}}$ (=64) transmitting and $\mathrm{N}_{\mathrm{r}}(=8)$ receiving antennas are arranged in uniform linear array (ULA). Such MIMO channel has limited scattering with $\mathrm{Lu}(=6)$ scatterers. Each scatterer is assumed to contribute a single propagation path between the base station (BS) and mobile station (MS). The geometrical channel model $\mathrm{H} \in \mathrm{C}_{\mathrm{r}}^{\mathrm{N} \times \mathrm{N}}$ can be written as:

$$
H=\sqrt{\frac{N_{t} N_{r}}{\rho L_{u}} \sum_{l=1}^{L_{u}} \alpha_{u, l} a_{M S}\left(\theta_{u, l}\right) a_{B S}^{*}\left(\varphi_{u, l}\right)}
$$

where, $\alpha_{u, l}$ is the complex gain of the lth path including the path loss, $\rho$ is the path loss between base station (BS) and mobile station (MS). The variable $\theta_{u, l}$ and $\varphi_{u, l} \in[0,2 \pi]$ are the lth path's angle of arrival and departure (AoAS/AoDs) respectively. Finally, $a_{B S}\left(\varphi_{u, l}\right)$ an $\mathrm{d} a_{M S}\left(\theta_{u, l}\right)$ are the antenna array response vectors of the BS and MS respectively.

With available knowledge of the geometry of uniform linear antenna arrays, ${ }_{B S}\left(\varphi_{u, l}\right)$ is defined as:

$$
a_{B S}\left(\varphi_{u, l}\right)=\frac{1}{\sqrt{N_{t}}}\left[1, e^{j \frac{2 \pi}{\lambda} d \sin \left(\varphi_{u, l}\right), \ldots \ldots \ldots \ldots \ldots . .} e^{j\left(N_{t}-1\right) \frac{2 \pi}{\lambda} d \sin \left(\varphi_{u, l}\right)}\right]^{T}
$$

and

$$
a_{M S}\left(\theta_{u, l}\right)=\frac{1}{\sqrt{N_{r}}}\left[1, e^{j \frac{2 \pi}{\lambda} d \sin \left(\theta_{u, l}\right), \ldots . .} e^{j\left(N_{r}-1\right) \frac{2 \pi}{\lambda} d \sin \left(\theta_{u, l}\right)}\right]^{T}
$$

where, $\lambda$ is the signal wavelength and $d$ is the distance between two consecutive antenna elements.

The MIMO channel $\mathbf{H}$ is further normalized to get its Fresenius norm value-

$$
\hat{H}=S \odot H
$$

where, $\odot$ is indicative of Hadamard product, $S$ the $8 \times 64$ sized matrix whose each element is inverse of magnitude of each complex element of $\mathrm{H}$. The squared Frobenius norm of the normalized channel matrix $\hat{H}$ is given by $[7,8]$

$$
\left[\left\|\hat{H}_{F}\right\|^{2}\right]=N_{t} N_{r}
$$

\subsection{Digital Precoding}

Digital precoding is generally used to control both the phases and amplitudes of the original signals to cancel interferences in advance. In consideration of designing digital precoding for single-user mmWave massive MIMO system, it is assumed that the base station (BS) employs $\mathrm{N}_{\mathrm{t}}$ antennas to 
simultaneously transmit $\mathrm{N}_{\mathrm{r}}$ data streams to a user with $\mathrm{N}_{\mathrm{r}}$ antennas $\left(\mathrm{N}_{\mathrm{r}}<\mathrm{N}_{\mathrm{t}}\right)$. The BS applies an $\mathrm{N}_{\mathrm{t}} \times \mathrm{N}_{\mathrm{r}}$ digital precoder $\mathrm{D}$ and the transmitted signal prior to $\mathrm{D} / \mathrm{A}$ conversion can be presented by-

$$
\mathrm{x}=\mathrm{Ds}
$$

where, $\mathrm{s}$ is the $\mathrm{N}_{\mathrm{r}} \times 1$ original signal vector before precoding with normalized power as $\mathrm{E}\left(\mathrm{ss}^{\mathrm{H}}\right)=\left(1 / \mathrm{N}_{\mathrm{r}}\right) \mathrm{I}_{\mathrm{Nr}}$, To meet up the total transmit power, D satisfies

$$
\|D\|_{F}^{2}=\operatorname{trace}\left(D D^{T}\right)=N_{r}
$$

In terms of geometrical channel presented in Equation (4), the digital precoder is given by-

$$
D=\sqrt{\frac{N_{r}}{\operatorname{tr}\left(F F^{H}\right)} F}
$$

where, $\mathrm{F}=\hat{H}^{H}$

\subsection{Signal Detection Scheme}

In our $8 \times 64$ simulated system, the received signal in terms of D/A converted transmitted signal $\hat{x}$, fading channel $\hat{H}$ and white Gaussian noise $\hat{n}$ with a variance $\sigma_{\mathrm{n}}{ }^{2}$ can be written as:

$$
\hat{\mathbf{y}}=\hat{\mathbf{H}} \hat{\mathbf{x}}+\hat{n}
$$

In Minimum mean square error (MMSE) based signal detection scheme, the MMSE weight matrix is given by-

$$
W_{M M S E}=\left(\hat{H}^{H} \hat{H}+\sigma_{n}^{2} I\right)^{-1} \hat{H}^{H}
$$

where, (.) $)^{\mathrm{T}}$ is applicable to all cases to indicate complex conjugate (Hermitian) transformation and the detected desired signal from the transmitting antenna is given by

$$
\tilde{x}_{M M S E}=W_{M M S E} \hat{y}
$$

In Zero-Forcing (ZF) scheme, the ZF weight matrix is given by-

$$
W_{Z F}=\left(\hat{H}^{H} \hat{H}\right)^{-1} \hat{H}^{H}
$$

and the detected desired signal from the transmitting antenna is given by [9]

$$
\tilde{x}_{Z F}=W_{Z F} \hat{y}
$$

In LR based linear signal detection scheme, the channel matrix $\hat{\mathbf{H}}$ is decomposed into unimodular matrix $\hat{U}$ and orthogonal matrix $\hat{G}$ such that

$$
\hat{\mathrm{H}}=\hat{G} \hat{U}
$$

The unimodular matrix $\hat{U}$ is estimated using the following relation:

$$
\hat{U}=\hat{\hat{\mathbf{H}}} \hat{\mathbf{H}}^{T}
$$

where, the matrix $\hat{\hat{\mathbf{H}}}$ is the Moore-Penrose pseudo-inverse of matrix $\hat{\mathbf{H}}$. The equation (14) can be rewritten as:

$$
\hat{U}^{T} \hat{G}=\hat{\mathbf{H}}^{T}
$$

From equation (16), orthogonal matrix $\hat{G}$ is estimated as:

$$
\hat{G}=\left(\hat{U}^{T}\right)^{-1} \hat{H}^{T}
$$

The LR-based linear detection weight matrix, $\hat{W}$ is given by-

$$
\hat{W}^{T}=\left(\hat{G}^{T} \hat{G}\right)^{-1} \hat{G}^{T}
$$

the detected desired signal from the transmitting antenna is given by $[9,10]$

$$
\tilde{x}_{L R}=(\hat{U})^{-1} \hat{W}^{T} \hat{y}
$$

\subsection{Repeat and Accumulate Channel Coding}

The RA is a powerful modern error-correcting channel coding scheme. In such scheme, all the extracted binary bits from the color image has been arranged into a single block and the binary bits of the such block is repeated 2 times and rearranged into a single block containing binary data which is double of the number of input binary data.

\section{6. (3, 2) SPC Channel Coding}

In SPC channel coding, the transmitted binary bits are rearranged into very small code words consisting of merely two consecutive bits. In such coding, $(3,2)$ SPC code is used with addition of a single parity bit to the message $u=[u 0, u 1]$ so that the elements of the resulting code word $\mathrm{x}=[\mathrm{x} 0, \mathrm{x} 1, \mathrm{x} 2]$ are given by $\mathrm{x} 0=\mathrm{u} 0, \mathrm{x} 1=\mathrm{u} 1$ and $\mathrm{x} 2=\mathrm{u} 0$ 田 $\mathrm{u} 1$ [11] where, $\boxplus$ denotes the sum over GF (2)

\subsection{LDPC Channel Coding}

The low-density parity-check (LDPC) code known as Gallager code was invented as early as 1962 . Such code is an linear block code with its parity-check matrix $\mathrm{H}$ containing only a few 1 's in comparison to 0 's. The parity-check matrix $\mathrm{H}_{\mathrm{p}}$ used in this present study is $64 \times 128$ sized. In LDPC channel coding, the input binary data are rearranged into blocks with each block containing 64 binary bits. For each individual block, a $1 \times 128$ matrix sized code word $\mathrm{c}$ is produced. Its first 64 bits are the parity bits and the last 64 bits are the information bits. In LDPC channel decoding, iterative Log Domain Sum-Product LDPC decoding technique is implemented to operate alternatively on the bit nodes and the check nodes to find the most likely code word $\mathrm{c}$ that satisfies the condition $\mathrm{cH}_{\mathrm{p}}^{\mathrm{T}}=0$ [12]. In such decoding scheme, the log-likelihood ratio (LLR) of transmitted code ward $\mathrm{c}$ is estimated from received bit sequence $\mathrm{rx}_{\mathrm{i}}$ converted from $(0 / 1)$ format into (-1/1) format and passed through AWGN channel 
of noise variance of $\mathrm{N} 0$ as:

$$
\mathrm{Lc}_{\mathrm{i}}=-4 \quad \mathrm{rx}_{\mathrm{i}} / \mathrm{N} 0
$$

where, $\mathrm{i}=1,2,3$

The mathematical formulations presented in this section are based on MATLAB source codes available in the website at [13] for iterative Log Domain Sum-Product LDPC decoding algorithm. However, considering all 128 sampled values from Equation (20), a $64 \times 128$ sized [LCI] matrix is formed with identical sampled values at its each of 64 rows. In perspective of messages sending sent from bit nodes i to check nodes $j$, a $64 \times 128$ sized [LQIJ] matrix is considered which is formed from the element wise product of two matrices $\left[\mathrm{H}_{\mathrm{p}}\right]$ and $[\mathrm{LCI}]$ as:

$$
[\mathrm{LQIJ}]=[\mathrm{Hp}] \odot[\mathrm{LCI}]
$$

Two useful parameters, $\Phi$ and $\beta_{\mathrm{ij}}$ are estimated from the matrix [LQIJ] using the following relation:

$$
\Phi \triangleq \operatorname{sign}[\mathrm{LQIJ}] \quad \beta_{\mathrm{ij}} \triangleq|\mathrm{LQIJ}|
$$

Initially, a $64 \times 128$ sized [LRJI] matrix is considered as null matrix. In horizontal stepping for finding non zero in the column of $\mathrm{H}_{\mathrm{p}}$ matrix, the Pibetaij parameter values at the position $(\mathrm{r}, \mathrm{l})$ are estimated using the relation:

$$
\text { Pibetaij(r,l) }=\ln \left[\left(\exp \left(\beta_{\mathrm{ij}}(\mathrm{r}, \mathrm{l})+1\right) /\left[\left(\exp \left(\beta_{\mathrm{ij}}(\mathrm{r}, \mathrm{l})-1\right)\right]\right.\right.\right.
$$

At each position of non zero element, new values Pibetaij(i,c1) are estimated from the summation of all column wise Pibetaij values previous Pibetaij value at that position where, $\mathrm{i}=1,2, \ldots \ldots .64, \mathrm{c} 1$ is the non zero elemental position in the column for a row identified by $i$. With estimated values of Pibetaij(i,c1), PiSum(i,c1) are estimated as:
PiSum $(\mathrm{i}, \mathrm{c} 1)=\ln [(\exp ($ Pibetaij(i,c1) +1$) /[(\exp ($ Pibetaij(i,c1)-1) $(23)$

Similarly, another parameter prodOf $(i, c 1)$ values are estimated from the product of all column wise multiplied ब values with of value at that position. The previously considered [LRJI] matrix is upgraded through inserting the parameter Lrji $(\mathrm{i}, \mathrm{c} 1)$ values as:

$$
\operatorname{Lrji}(\mathrm{i}, \mathrm{c} 1)=\operatorname{prodOf}(\mathrm{i}, \mathrm{c} 1)^{*} \operatorname{PiSum}(\mathrm{i}, \mathrm{c} 1)
$$

In vertical stepping for finding non zero in the row of $\mathrm{H}_{\mathrm{p}}$ matrix, the Lqij parameter values at the position $(\mathrm{r} 1, \mathrm{j})$ are updated using the relation:

$$
\operatorname{Lqij}(r 1, j)=\operatorname{Lc}_{\mathrm{i}}+\operatorname{sum}(\operatorname{Lrji}(r 1, \mathrm{j}))-\operatorname{Lrji}(r 1, \mathrm{j})
$$

where, $i=1,2 \ldots \ldots \ldots 128, j=1,2 \ldots \ldots \ldots 128$

Finally, a new parameter value is estimated as:

$$
\mathrm{LQi}=\mathrm{Lc}_{\mathrm{i}}+\operatorname{sum}(\operatorname{Lrji}(\mathrm{r} 1, \mathrm{j}))
$$

If LQi is less than zero, the transmitted bit is 1 , otherwise the transmitted bit is 0 .

\subsection{D Median Filtering}

2D median filtering is widely used as an effective technique for removing various types of noises (salt and pepper and Gaussian) from noise contaminated image. In such filtering operation, the pixel values in the neighborhood window are generally ranked according to intensity and the middle value (the median) becomes the output value for the pixel under evaluation. In this paper, 2D Median Filtering scheme with a $3 \times 3$ neighborhood windowing mask is preferably used to make sorting of all the pixel values within the window and finding the median value and replacing the original pixel value with the median value [14].

\section{System Description}

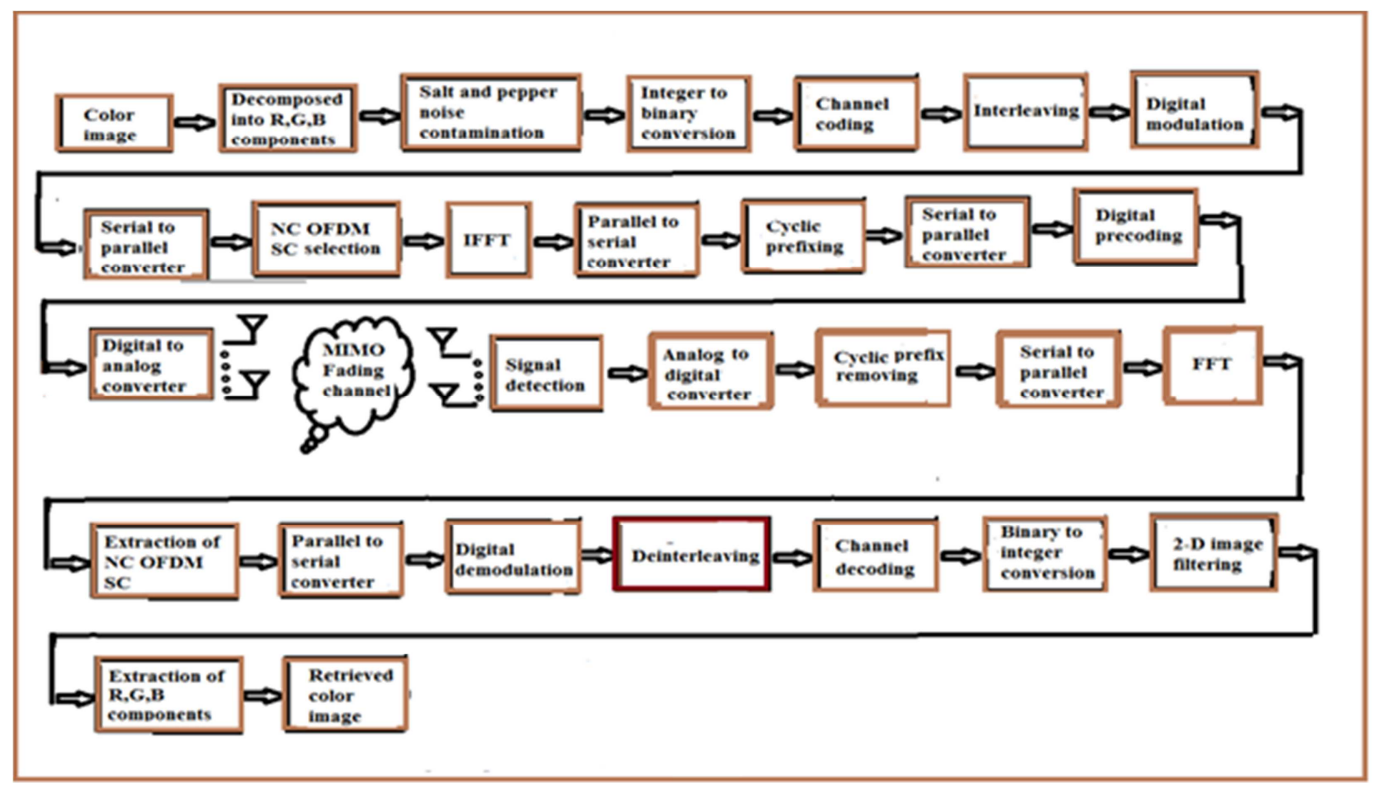

Figure 1. Block diagram of a Single-user Digitally Precoded mmWave NC-OFDM Wireless Communication System. 
The simulated single-user digitally precoded mmWave non-contiguous orthogonal frequency division multiplexing wireless communication system is presented in Figure 1. A RGB color image with 96 pixels (width) $\times 96$ pixels (height) is processed in such simulated system. It is converted into their respective three Red, Green and Blue components with each component is of also 96 pixels $\times 96$ pixels in size The pixel integer values [0-255] are contaminated with salt and pepper noise and the noise converted pixel integer values converted into 8 bits binary form and channel coded and interleaved and digitally modulated using 256-QAM [15]. The digitally modulated complex symbols are the serial-to-parallelly converted and fed into NC-OFDM subcarrier selection section and subsequently processed for multicarrier modulation, parallel to serial conversion, Cyclic prefixing and serial to parallel conversion for data transmission in eight streams. The complex data are fed into digital precoder, digital to analog conversion and eventually sent up from each of 64 transmitting antennas. In receiving section, all the transmitted signals are detected with linear signal detection schemes and the detected signals are analog to digitally converted and processed for cyclic prefixing removing, multicarrier demodulation, extraction of desired NC-OFDM subcarriers, parallel to serial conversion, digital demodulation, deinterleaving, channel decoding, binary to integer conversion, 2D image filtering, reconstruction of Red, Green and Blue components and eventually color image retrieving.

\section{Result and Discussion}

In this section, simulation results using MATLAB R2014a are presented to illustrate the significant impact of various types of signal detection techniques on performance evaluation of a single-user digitally precoded mmWave NC-OFDM wireless communication system in terms of bit error rate (BER) on color image transmission. The rate of noise contamination rate for transmitted color image is $5 \% \mathrm{viz}$. 461 pixels out of 9216 pixels are contaminated with impulsive noise for 96 pixels $\times 96$ pixels sized Red, Green and Blue components of the color image. In perspective of higher order digital modulation, 256-QAM has been preferably used. However, it has also been assumed that the channel state information (CSI) of the geometrically estimated mmWave large MIMO fading channel is available at the receiver and the fading channel coefficients are constant during simulation. The proposed model is simulated to evaluate the system performance with considering the following parameters presented in the Table 1.

Table 1. Summary of the Simulated Model Parameters.

\begin{tabular}{ll}
\hline Parameters & Types \\
\hline Data Type & Color image \\
Image Size & $(96 \times 96 \times 3)$ pixels \\
Carrier frequency & $28 \mathrm{GHz}$ \\
Path loss constant & 3 \\
Path loss, dB for carrier frequency wavelength $\lambda$ and transmitter-receiver distance, d & $-20 \log _{10}(\lambda / 4 \pi \mathrm{d})$ \\
Noise reduction image filter & $2 \mathrm{D}-\mathrm{Median}$ filter \\
Antenna configuration & $32 \times 256$ Large MIMO Channel \\
Channel Coding & LDPC, Repeat and Accumulate and (3, 2) SPC \\
LDPC Channel decoding & Log-domain sum product \\
Noise type & Impulse (Salt and pepper) and Gaussian \\
Digital Modulation & 256-QAM \\
Signal Detection Scheme & ZF, MMSE and LR based linear detection \\
SNR & 0 to 10 dB \\
Channel & AWGN and Rayleigh \\
\hline
\end{tabular}

It is quite obvious from visual inspection of the normalized power spectral density (PSD) curves of NC-OFDM transmission with rectangular windowing presented in Figure 2 that the mean out-of-band(OOB) interference power level is achieved at least $300 \mathrm{~dB}$ below the mean non-contiguous sub band power level with high and steep OOB power attenuation. The estimated normalized PSD for various frequencies is based on the consideration of sub carrier frequency spacing $(\Delta \mathrm{f})$ and sampling frequency (Fs) with values of $488.28 \mathrm{KHz}$ and $125 \mathrm{MHz}$ respectively for a FFT size is of 256(The bit rate is $1 \mathrm{Gbps}$ and the symbol rate is 125 Msymbols/sec). The graphical illustration presented in Figure 3 shows the system performance comparison in terms of Bit error rate (BER) Vs. SNR values under implementation of LDPC, Repeat and Accumulate and $(3,2)$ SPC channel coding schemes and ZF signal detection technique. Under typically assumed SNR value of $2 \mathrm{~dB}$, the estimated BER values are $0.1463,0.1553$ and
0.2256 in case of $(3,2)$ SPC, Repeat and Accumulate and LDPC channel coding schemes respectively. In such specifically considered SNR value, system performance improvement of $0.2593 \mathrm{~dB}$ and $1.8809 \mathrm{~dB}$ are achieved in $(3,2)$ SPC as compared to Repeat and Accumulate and LDPC channel coding techniques. At $10 \%$ BER, SNR gain of 0.1353 $\mathrm{dB}$ and $0.4018 \mathrm{~dB}$ are obtained in $(3,2) \mathrm{SPC}$ as compared to Repeat and Accumulate and LDPC channel coding techniques.

In Figure 4, it is noticeable that BER performances in case of implemented Repeat and Accumulate and $(3,2)$ SPC are not well discriminated from each other. For a typically assumed SNR value of $2 \mathrm{~dB}$, the estimated BER values are $0.1449 \mathrm{~dB}$ and $0.0 .2346 \mathrm{~dB}$ in case of Repeat and Accumulate and LDPC which implies a system performance improvement of $2.0926 \mathrm{~dB}$. At $10 \% \mathrm{BER}$, the SNR gain $0.0926 \mathrm{~dB}$ is obtained in Repeat and Accumulate as compared to LDPC channel coding technique. 


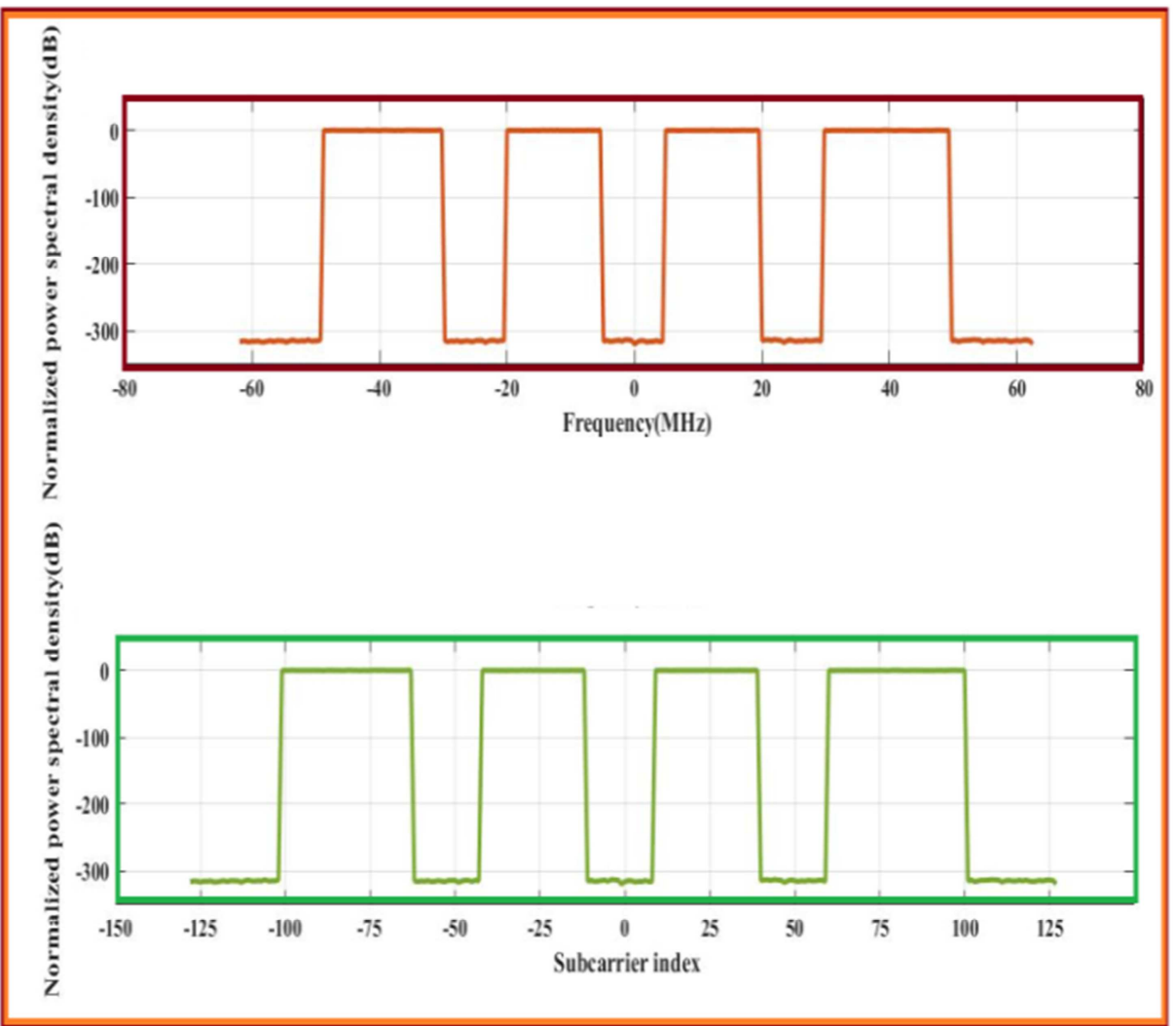

Figure 2. Comprehensive graphical illustration showing estimated normalized power spectral density (PSD) for the NC-OFDM transmission signal at various subcarrier index and frequencies relative to center frequency (shown as zero).

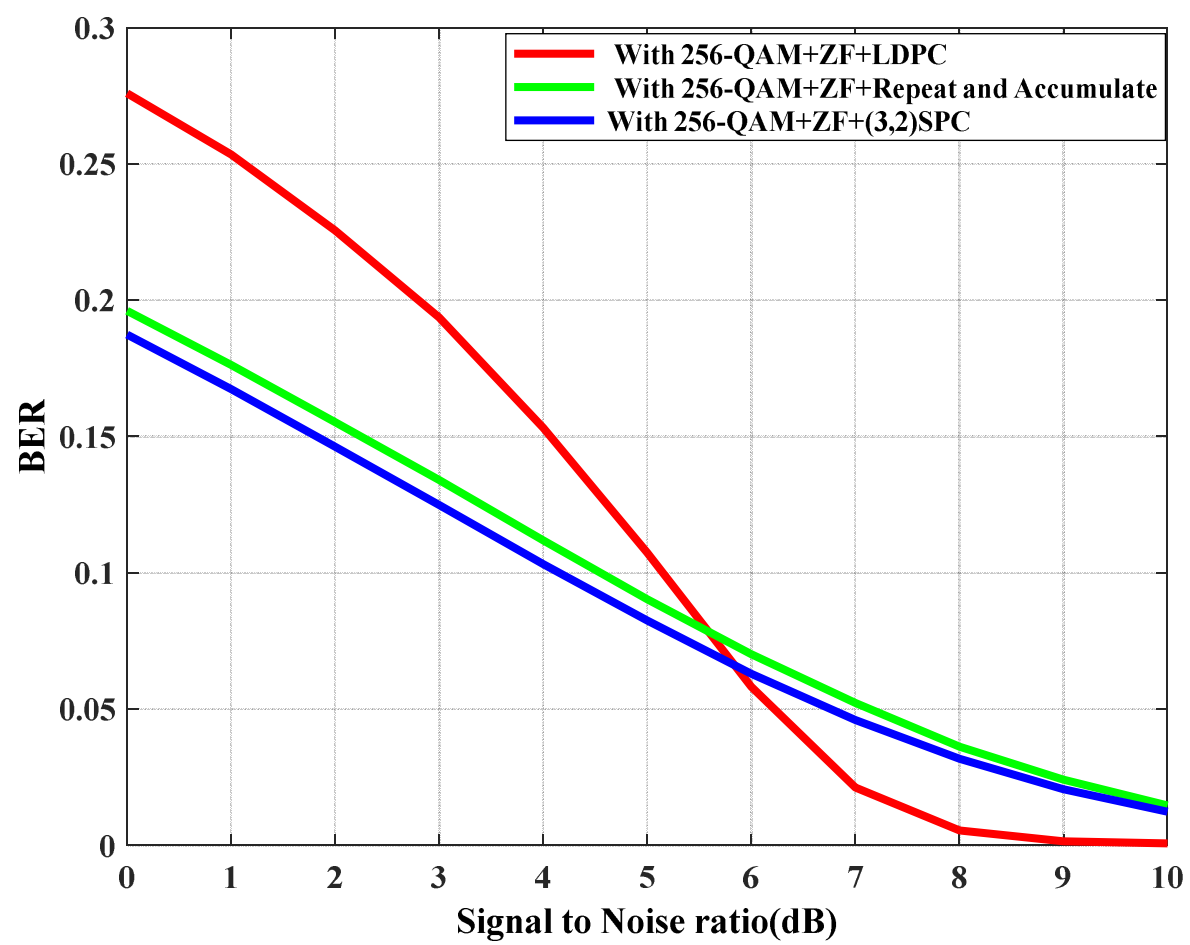

Figure 3. BER performance of various channel encoded single-user digitally precoded mmWave NC-OFDM wireless communication system under utilization of 256-QAM and ZF signal detection technique 


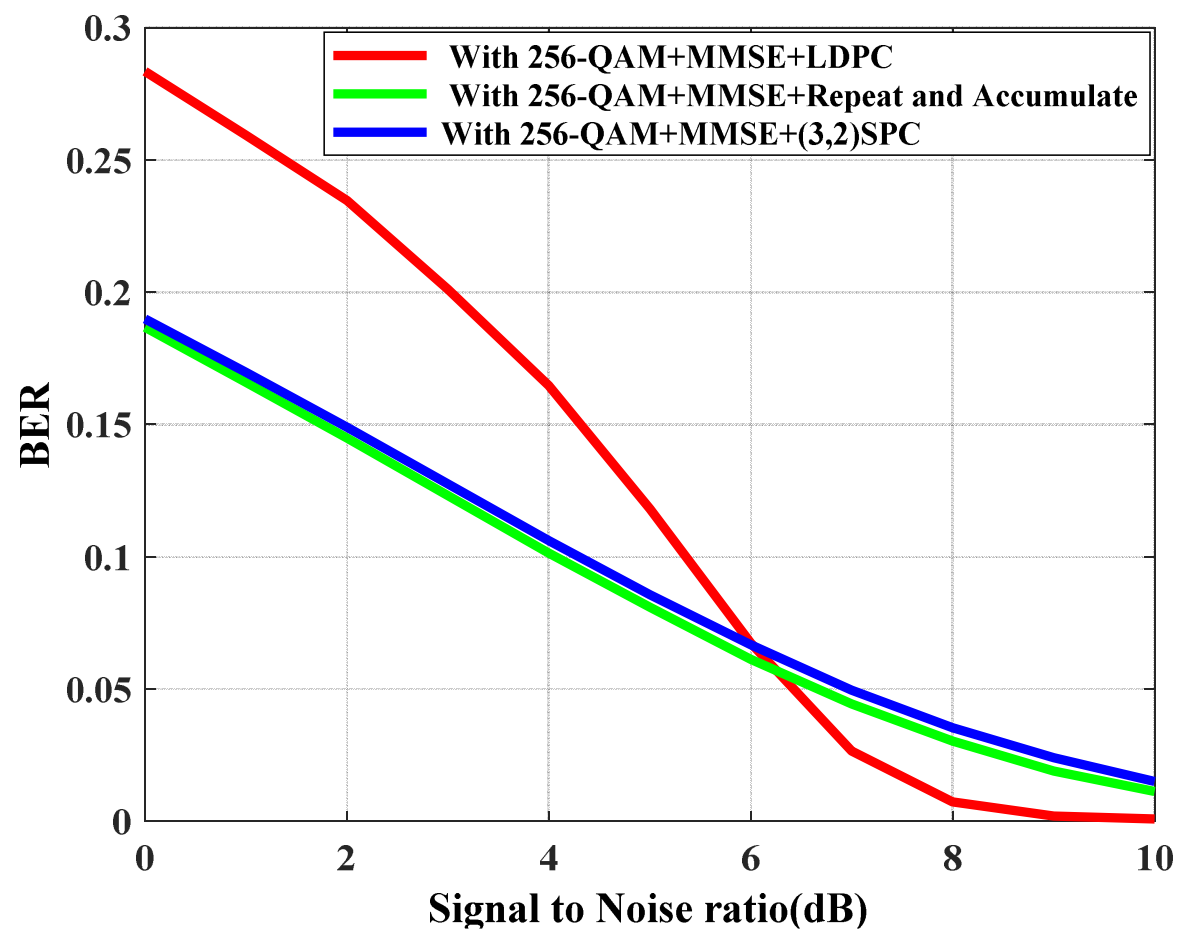

Figure 4. BER performance of various channel encoded single-user digitally precoded mmWave NC-OFDM Wireless communication system under utilization of 256-QAM and MMSE signal detection technique.

Figure 5 represents the BER performances under implementation of LDPC, Repeat and Accumulate and $(3,2)$ SPC channel coding schemes for LR based signal detection technique. Under consideration of SNR value of $2 \mathrm{~dB}$, the estimated BER values are $0.1549,0.1477$ and 0.2271 for $(3,2)$ SPC Repeat and Accumulate and LDPC channel coding schemes respectively. In such specifically considered SNR value, system performance improvement of $0.2067 \mathrm{~dB}$ and $1.8684 \mathrm{~dB}$ are achieved in Repeat and Accumulate as compared to $(3,2)$ SPC and LDPC channel coding schemes. At $10 \%$ BER, the SNR gain $0.3120 \mathrm{~dB}$ and $1.2500 \mathrm{~dB}$ are obtained in Repeat and Accumulate in comparison with $(3,2)$ SPC and LDPC channel coding schemes.

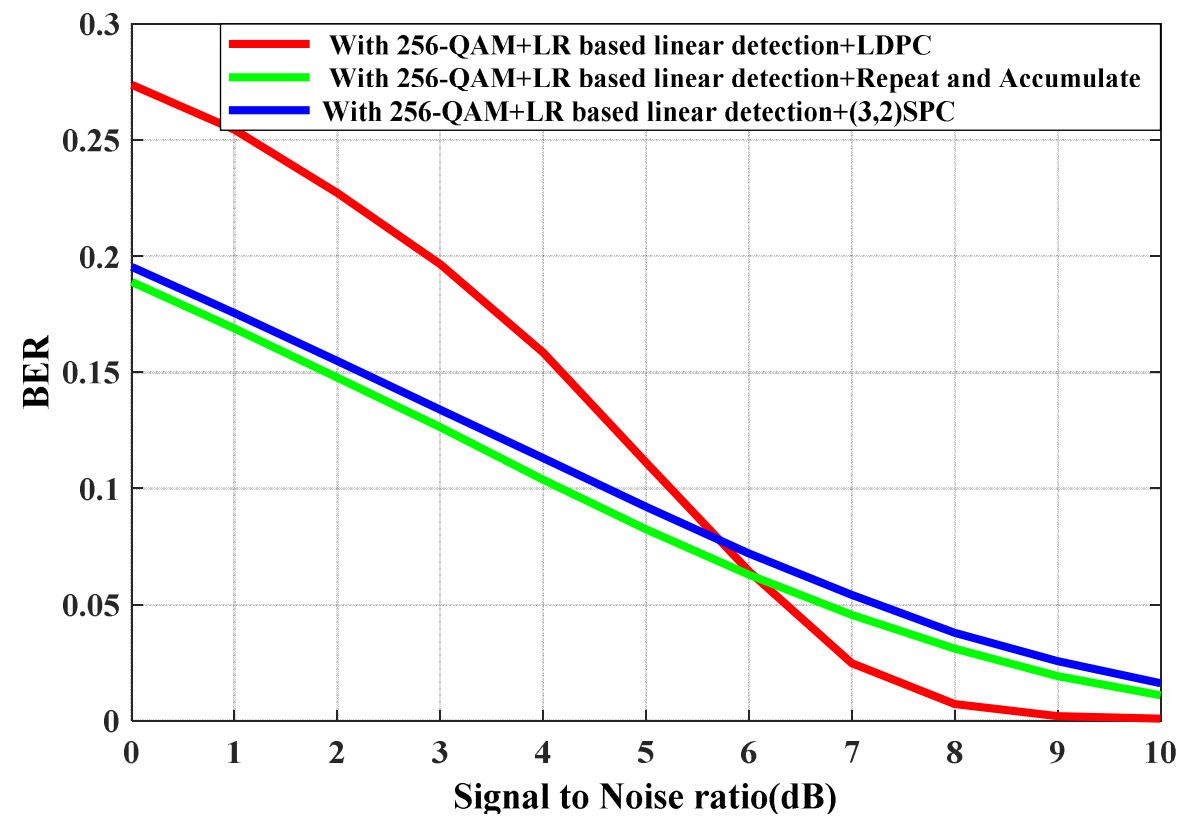

Figure 5. BER performance of various channel encoded single-user digitally precoded mmWave NC-OFDM wireless communication system under utilization of 256-QAM and LR based signal detection technique.

On critical observation at various images presented in Figure 6 , it is observable that the quality of the retrieved images improves with the increase in SNR values. The impact of 2-D filtering technique on improvement of 
retrieved image is reasonably acceptable. In Figure 7, it is quite obvious that the pixel values of the original color image have comparatively lower pixel values. Distributions of pixel values are changing due to salt and pepper and AWGN noise contamination. In case of higher SNR value preferably $10 \mathrm{~dB}$ and filtered image, the presented histograms get resemblance as to original image. In Figure 8, 3-dimensional graphical illustration showing transmitted, salt and pepper noise contaminated and retrieved color images with and without filtering have been presented to justify the suitability of our proposed single-user digitally precoded mmWave non-contiguous orthogonal frequency-division multiplexing wireless communication system.

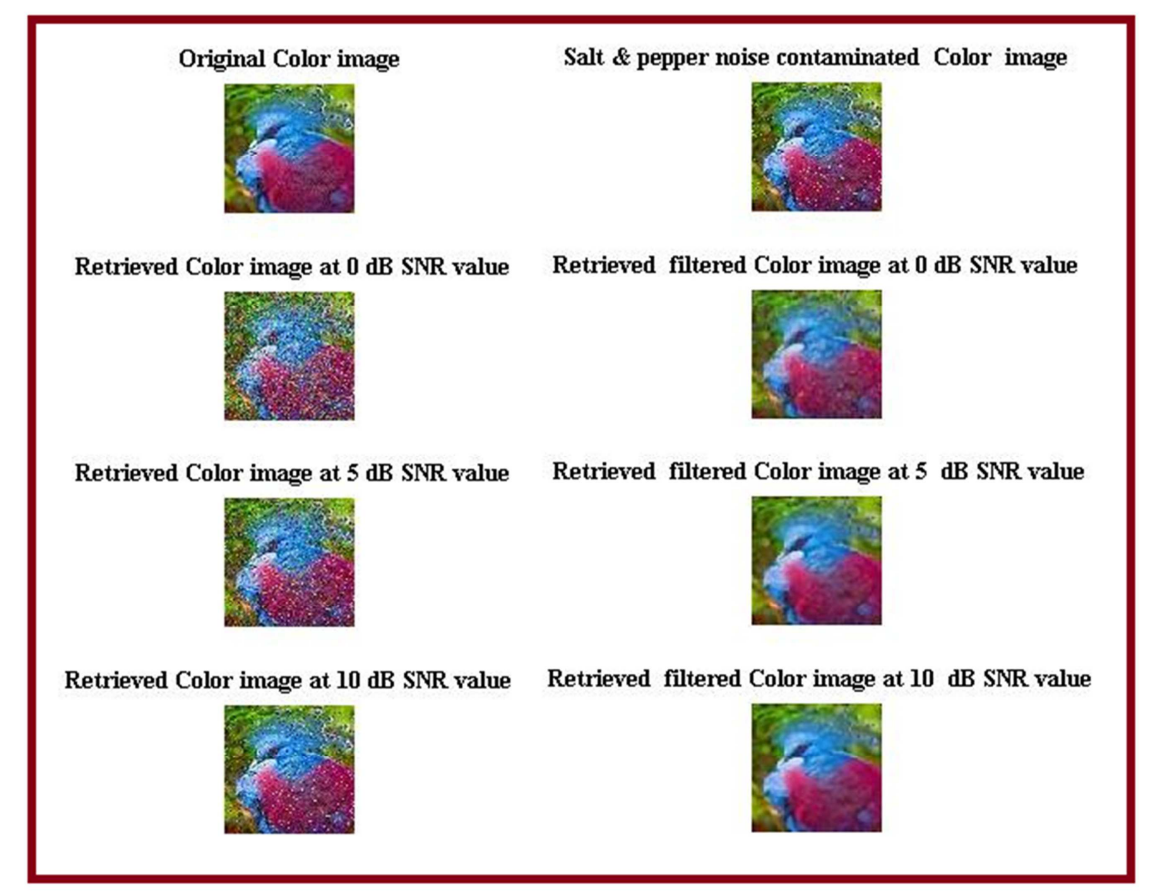

Figure 6. Transmitted, Pepper and Salt noise contaminated and retrieved color images with and without filtering in a single-user digitally precoded mmWave NC-OFDM wireless communication system.

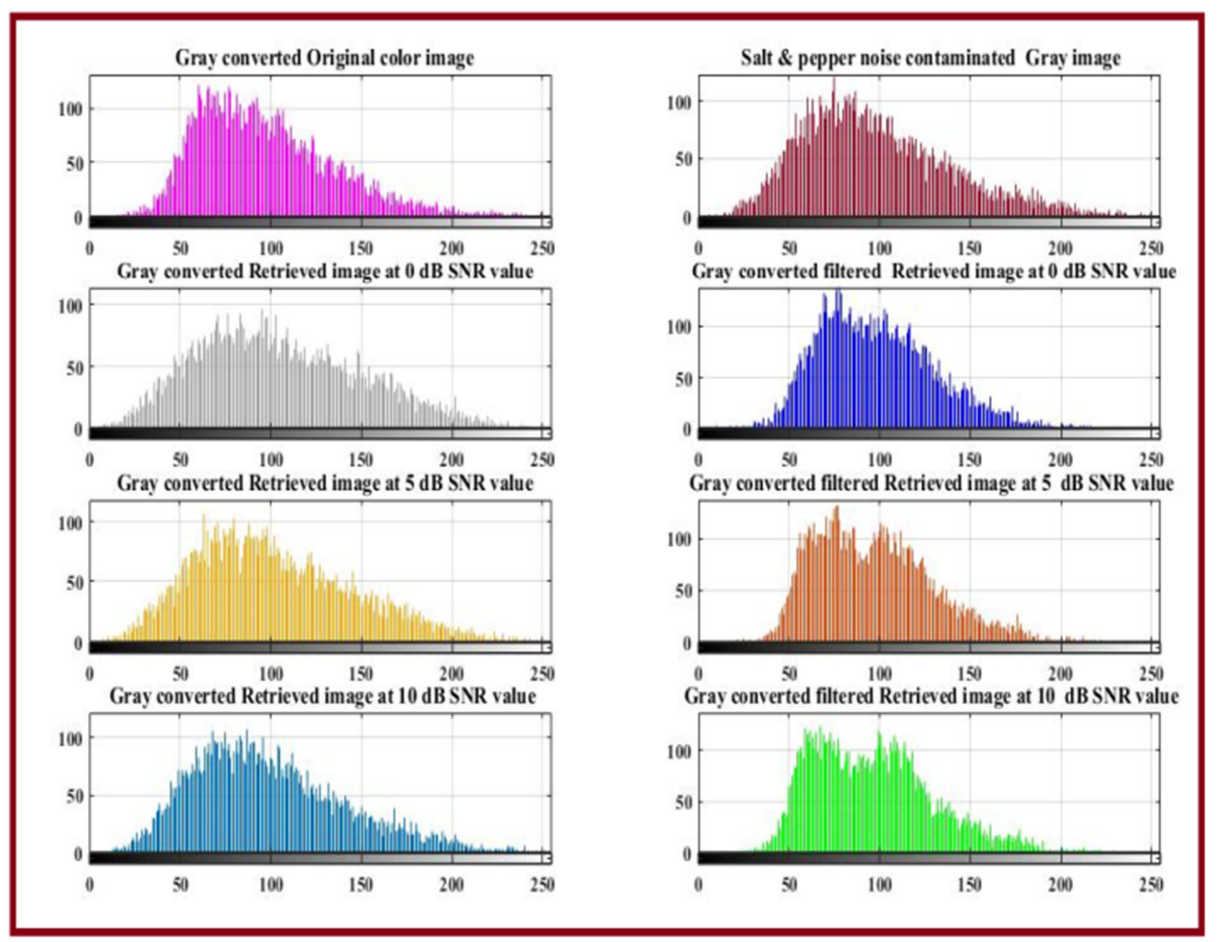

Figure 7. Histogram of RGB to Gray converted transmitted, Pepper and Salt noise contaminated and retrieved color images with and without filtering in a single-user digitally precoded NC-OFDM wireless communication system. 


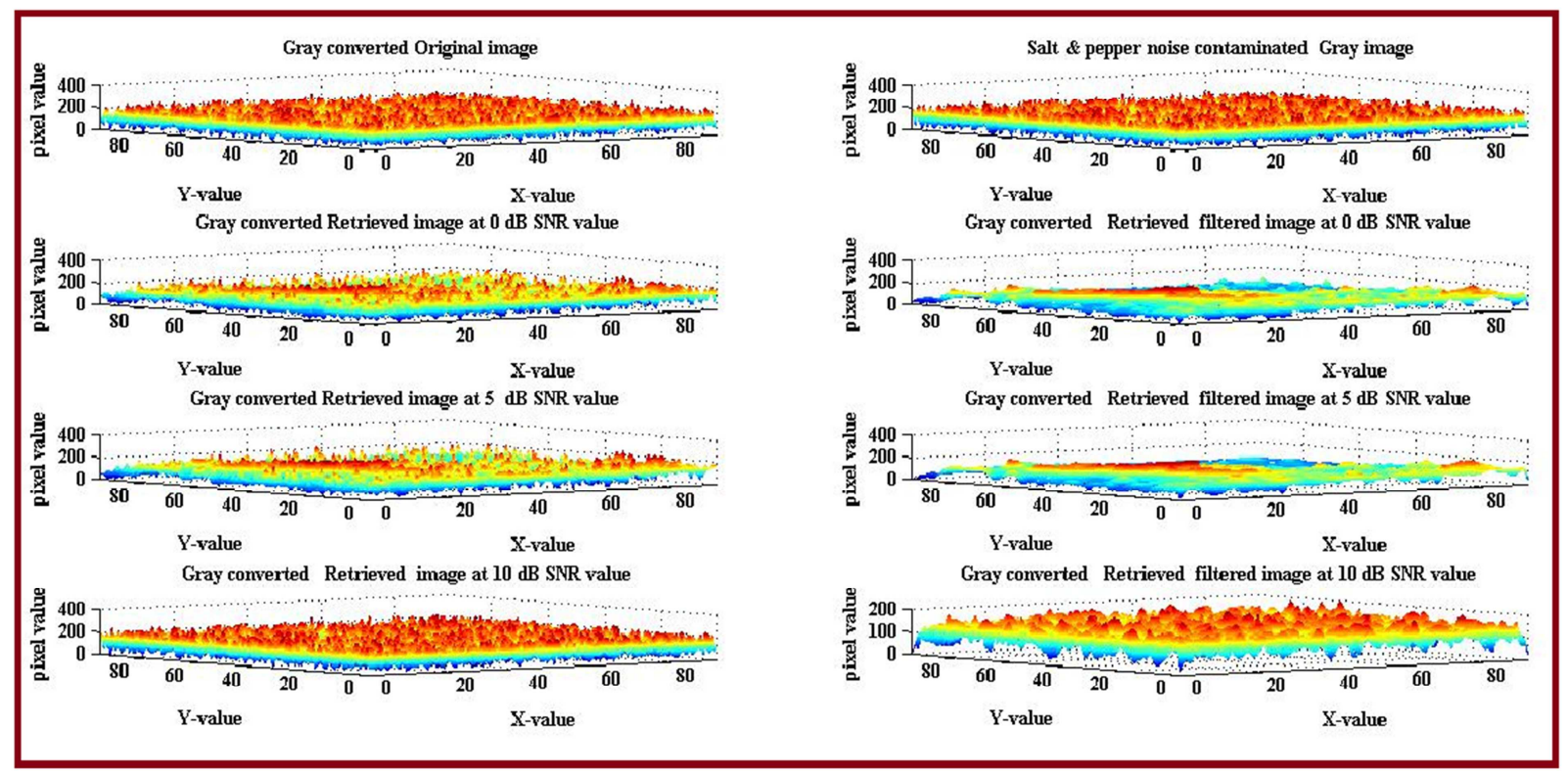

Figure 8. 3-Dimensional Graphical illustration showing RGB to Gray converted transmitted, Pepper and Salt noise contaminated and retrieved color images with and without filtering single-user digitally precoded mmWave NC-OFDM wireless communication system.

\section{Conclusion}

In this paper, the performance of single-user digitally precoded mmWave non-contiguous orthogonal frequency-division multiplexing (NC-OFDM) wireless communication system has been investigated on color image transmission under utilization of various channel coding and signal detection techniques. From the simulation results, it can be concluded that the presently considered single-user digitally precoded mmWave non-contiguous orthogonal frequency-division multiplexing (NC-OFDM) wireless communication system shows satisfactory performance with higher order digital modulation under implementation of MMSE signal detection and Repeat and Accumulate channel coding technique.

\section{References}

[1] Hanna Bogucka, Adrian Kliks and Paweł Kryszkiewicz, 2017: Advanced Multicarrier Technologies for Future Radio Communication 5G and Beyond, John Wiley and Sons, Inc, USA.

[2] Damoon Shahbaztabar and Hamid Farrokhi, 2014: Designing non-contiguous orthogonal frequency division multiplexing transceiver based on wavelet transform and removable cyclic prefix for spectrum sharing in cognitive radio systems, IET Communications, vol. 8, no.9, pp. 1508-1517.

[3] Miin-Jong Hao, Member and Chiu-Hsiung Lai, 2015: Precoding for PAPR reduction and sidelobe suppression for NC-OFDM based cognitive radio systems in International Symposium on Intelligent Signal Processing and Communication Systems (ISPACS), pp.542-547.

[4] Atif Elahi, Mehreen Atif, Ijaz Mansoor Qureshi and Noor Gul, 2017: Interference Reduction in Cognitive Radio Networks Using Genetic and Firefly Algorithms in International Conference on Communication, Computing and Digital Systems (C-CODE), pp.96-100.
[5] Shaik Yasmin Fathima, Muhammad Zia Ur Rahman, Murali Krishna, Shakira Bhanu, Mirza Shafi Shahsava, 2017: Side Lobe Suppression in NC-OFDM Systems Using Variable Cancellation Basis Function, IEEE Access, vol.5, pp.94159421.

[6] Shahid Mumtaz, Jonathan Rodriguez and Linglong Dai, 2017: mmWave Massive MIMO A Paradigm for 5G, Academic Press, an imprint of Elsevier Inc, United kingdom.

[7] Ahmed Alkhateeb, Omar El Ayach, Geert Leus, and Robert W. Heath Jr, 2013: Hybrid Precoding for Millimeter Wave Cellular Systems with Partial Channel Knowledge in IEEE workshop on Information Theory and Applications (ITA) pp.1-5.

[8] Weiheng Ni, Xiaodai Dong, and Wu-Sheng Lu, 2015: Near-Optimal Hybrid Processing for Massive MIMO Systems via Matrix Decomposition, pp.1-9, cited as: arXiv: $1504.03777 \mathrm{v} 1$ [cs.IT].

[9] Lin Bai and Jinho Choi, 2012: Low Complexity MIMO Detection Springer Science+Business Media, LLC, New York, USA.

[10] Ying Hung Gan, Cong Ling, and Wai Ho Mow, 2006: Complex Lattice Reduction Algorithm for Low-Complexity MIMO Detection, pp.1-9, cited as: arXiv:cs/0607078 [cs.DS].

[11] Giorgio M. Vitetta, Desmond P. Taylor, Giulio Colavolpe, Fabrizio Pancaldi and Philippa A. Martin, 2013:Wireless Communications Algorithmic Techniques. John Wiley and Sons Ltd, United Kingdom.

[12] Yuan Jiang, 2010:A Practical Guide to Error-Control Coding Using MATLAB, Artech House, Norwood, MA, USA.

[13] Bagawan Sewu Nugroho, LDPC Code Using MATLAB and C MEX.

[14] OgeMarques, 2011: Practical Image and Video Processing Using MATLAB, John Wiley and Sons, New Jersey, USA.

[15] Theodore S Rappaport, 1996: Wireless Communications Principles and Practice, Second Edition. 